Pacific Journal of Mathematics

TYpESETS AND COTYPESETS OF RANK-2 TORSION FREE David Marion ARNold and Charles IRvin Vinsonhai 


\title{
TYPESETS AND COTYPESETS OF RANK-2 TORSION FREE ABELIAN GROUPS
}

\author{
D. Arnold And C. Vinsonhaler
}

\begin{abstract}
A sufficient condition is given for a set of types to be the typeset of a rank-2 group, strengthening all previous results on this subject. A correct version of a theorem of Schultz on types and cotypes is provided, along with a variety of other results on typesets and cotypesets of rank-2 groups. Numerous examples are included.
\end{abstract}

Beaumont-Pierce [3], in 1961, posed the problem of finding necessary and sufficient conditions for a set of types to be the typeset of a rank-2 torsion free abelian group. They also, among other things, solved the problem in case the given set of types is finite. Koehler [10], in 1964, extended some of these results. Dubois [5] and [6], 1965 and 1966, used basic analytic number theory techniques to give some necessary and some sufficient conditions for a set of types to be realized as the typeset of a rank-2 torsion free abelian group.

Ito [9], in 1975, gave a sufficient condition for the realization of a set of types, which is easily seen to be equivalent to a sufficient condition of Dubois [5], Theorem 1. Ito's construction, however, is somewhat easier to understand, the group being given as a homomorphic image of a completely decomposable group rather than as a subgroup of the direct sum of two copies of the $Z$-adic integers.

Schultz [11], in 1978, claimed to have given necessary and sufficient conditions on two sets of types $S_{1}$ and $S_{2}$ such that there is a rank-2 group $A$ with typeset $(A)=S_{1}$ and $\operatorname{cotypeset}(A)=S_{2}$. A counterexample to this theorem is given by Vinsonhaler-Wickless [12] (also see Example 1.6). Vinsonhaler-Wickless [12] also give some simple necessary and sufficient conditions for a set of types to be realized as the cotypeset of a rank-2 group.

The theme of this paper is to examine Dubois' results from the point of view of Ito's construction. This point of view leads to:

THEOREM. Let $S=\left\{\tau_{1}, \tau_{2}, \ldots\right\}$ be a set of types with $\inf \left(\tau_{l}, \tau_{\jmath}\right)=$ type $(Z)$ whenever $\tau_{t} \neq \tau_{l}$. Assume that $\left\{\tau_{i} \mid \tau_{t}\right.$ is very large $\}$ has no snarls in $S$.

(a) There is a rank-2 group $A$ with typeset $(A)=S$ iff either type $(Z) \in$ $S$ or else $S$ has an infinite subset with no snarls in $S$; 
(b) Let $S^{\prime}=\left\{\sigma_{1}, \sigma_{2}, \ldots\right\}$ be another set of types. Then there is a rank-2 group $A$ with typeset $(A)=S$ and $\operatorname{cotypeset}(A)=S^{\prime}$ if and only if

(i) There is a type $\sigma_{0}=\sup \left\{\sigma_{i}, \sigma_{j}\right\}$ for $i \neq j$;

(ii) $\tau_{i} \leq \sigma_{0}$ for each $i$;

(iii) $\sigma_{i}=\sigma_{0}-\tau_{i}$ for each $i$; and

(iv) Either type $(Z) \in S$ or else $S$ has an infinite subset with no snarls in $S$.

Included as special cases are Dubois' theorems (Theorem 2.9(b) and Corollary 2.10); Koehler's results (Theorem 2.9(a)); Ito's theorem (Corollary 2.14(b) which includes the Beaumont-Pierce results), and a corrected version of Schultz's assertions (Corollary 2.11). Also included is a simplification of the proof of the Vinsonhaler-Wickless theorem (Theorem 3.1).

Of particular interest are the locally completely decomposable groups, discussed in \$4. If $A$ is a finite rank torsion free group then there are locally completely decomposable groups $B$ and $\epsilon$ with $B \subseteq A \subseteq C ; C / B$ torsion; $\operatorname{typeset}(A)=\operatorname{typeset}(B)$, and $\operatorname{cotypeset}(A)=\operatorname{cotypeset}(C)($ Theorem 4.1).

Section 5 is devoted to some open questions.

This paper is largely self-contained, and as a result partially expository, due to the complexity and the history of the problems considered. However, references for published results are given as well as numerous examples.

0. Notation and preliminaries. The basic properties of finite rank torsion free groups may be found in Fuchs [7]. Special notation used herein includes: if $h$ is a height sequence (characteristic) then $h(p)$ is the $p$ th entry for a prime $p ; \tau=[h]$ denotes the type of $h$, an equivalence class of height sequences; and write $h \in \tau$. If $A$ is torsion free and $a \in A$ then $h^{A}(a), h_{p}^{A}(a)$, and type ${ }_{A}(a)$ denote the height sequence, $p$-height, and type of $a$ in $A$, respectively. If $\operatorname{rank} A=1$ then $\operatorname{type}(A)=\operatorname{type}_{A}(a)$ for $0 \neq a \in A$.

If $\tau=[t]$ and $\sigma=[s]$ then $\inf \{\tau, \sigma\}$ and $\sup \{\tau, \sigma\}$ are defined by $[\min \{t, s\}]$ and $[\max \{t, s\}]$, respectively. For two types $\tau$ and $\sigma, \tau \leq \sigma$ iff there is $t \in \tau$ and $s \in \sigma$ with $t \leq s$. In this case $\sigma-\tau=[s-t]$, agreeing that $\infty-\infty=0$.

If $S$ is a subset of $A$ then $\langle S\rangle$ denotes the subgroup of $A$ generated by $S$ and $\langle S\rangle_{*}$ denotes the pure subgroup of $A$ generated by $S$.

Also assumed are some basic analytic number theory results as found, for example, in Hardy-Wright [8]. For a positive integer $n$, let $\pi(n)$ be the 
number of primes $\leq n$. Let $U=\{(r, s) \mid r, s \in Z, r \geq 0, \operatorname{gcd}(r, s)=1\}$ and $I_{n}=\{(r, s) \in U \mid \max \{r,|s|\} \leq n\}$. Denote the $i$ th prime by $p_{l}$.

\section{LEMMA 0.1.}

(a) (Chebyshev's Theorem.) There is $c_{1}>0$ and $c_{2}>0$ such that $c_{1} n / \log n<\pi(n)<c_{2} n / \log n$.

(b) $\lim _{n \rightarrow \infty} n / p_{n}=0$.

(c) $\left|I_{n}\right|=4 \Phi(n)$ where $\Phi(n)=\Sigma\{\phi(i) \mid 1 \leq i \leq n\}$ and $\phi(i)$ is the Euler $\phi$-function.

(d) $\phi(n)=3 n^{2} / \pi^{2}+O(n \log n)$

(e) $\lim _{n \rightarrow \infty}\left(\left|I_{n}\right|-\pi\left(2 n^{2}\right)\right)=\infty$.

Note that (b) follows from (a) since $n=\pi\left(p_{n}\right)<c_{2} p_{n} / \log p_{n}$ implies that $n / p_{n}<c_{2} / \log p_{n}$. Also (c) is a fairly routine counting argument, while (e) follows from (d) and (a).

As a consequence of (b), if $c$ is any constant then for sufficiently large $n, p_{n}>c n$.

1. Type sequences. A type sequence is a countably infinite sequence of types (repetition of types is permitted). Two type sequences $T$ and $T^{\prime}$ are equivalent, $T \approx T^{\prime}$, if one is a permutation of the other.

Let $A$ be a rank-2 group and let $A_{1}, A_{2}, \ldots$ be an indexing of the pure rank-1 subgroups of $A$. Define $\tau_{i}=\operatorname{typeset}\left(A_{i}\right)$ and $\sigma_{i}=\operatorname{type}\left(A / A_{l}\right)$ for each $i$. Then $T_{A}=\left(\tau_{1}, \tau_{2}, \ldots\right)$ and $C_{A}=\left(\sigma_{1}, \sigma_{2}, \ldots\right)$ are type sequences. Note that $T_{A}$ and $C_{A}$ are unique up to equivalence. $\operatorname{Define} \operatorname{typeset}(A)=$ $\left\{\tau_{l} \mid i \geq 1\right\}$ and $\operatorname{cotypeset}(A)=\left\{\sigma_{l} \mid i \geq 1\right\}$.

The following proposition is folklore.

Proposition 1.1. Let $A$ be a rank-2 group with $T_{A}=\left(\tau_{1}, \tau_{2}, \ldots\right)$ and $C_{A}=\left(\sigma_{1}, \sigma_{2}, \ldots\right)$.

(a) There is a type $\tau_{0}$ such that $\tau_{0}=\inf \left\{\tau_{l}, \tau_{j}\right\}$ for each $i \neq j$ and if typeset $(A)$ is finite then $\tau_{0}=\tau_{l}$ for some $i \geq 1$.

(b) There is a type $\sigma_{0}$ such that $\sigma_{0}=\sup \left\{\sigma_{l}, \sigma_{j}\right\}$ for each $i \neq j$ and if $\operatorname{cotypeset}(A)$ is finite then $\sigma_{0}=\sigma_{t}$ for some $i \geq 1$.

(c) $\tau_{t} \leq \sigma_{j}$ for each $i \neq j$ and $\tau_{0} \leq \sigma_{0}$.

(d) $\sigma_{l}-\tau_{j}=\sigma_{j}-\tau_{l}$ for each $i \neq j$ with $i \geq 0$ and $j \geq 0$.

(e) If $\tau_{0}=\operatorname{type}(Z)$ then $\sigma_{l}=\sigma_{0}-\tau_{l}$ for each $i$.

Proof. (a) If $i \neq j$ then $A /\left(A_{l} \oplus A_{l}\right)$ is torsion. Thus, for each $k$, $\tau_{k} \geq \inf \left\{\tau_{l}, \tau_{j}\right\}$. Consequently, if $k \neq l$ then $\inf \left\{\tau_{k}, \tau_{l}\right\} \geq \inf \left\{\tau_{l}, \tau_{j}\right\} \geq$ $\inf \left\{\tau_{k}, \tau_{l}\right\}$. Now assume that $\operatorname{typeset}(A)=\left\{\tau_{1}, \tau_{2}, \ldots, \tau_{n}\right\}$. If $j>n$ then 
$\tau_{0}=\inf \left\{\tau_{j}, \tau_{i}\right\}$ for each $1 \leq i \leq n$. But $\tau_{J}=\tau_{l}$ for some $1 \leq i \leq n$ so that $\tau_{0}=\tau_{i} \in \operatorname{typeset}(A)$.

The proof of $(b)$ is similar.

(c) follows from the fact that if $i \neq j$ then there is a monomorphism $A_{l} \rightarrow A / A_{\text {, }}$.

(d) First assume that $i \neq j$ are both non-zero. There is an exact sequence $0 \rightarrow A_{l} \rightarrow A / A_{j} \rightarrow A /\left(A_{i} \oplus A_{j}\right) \rightarrow 0$. Choose $0 \neq a_{i} \in A_{i}$ and $x_{j} \in A / A_{j}$ with $a_{i} \rightarrow x_{j}$. Then

$$
0 \rightarrow A_{i} / Z a_{i} \rightarrow\left(A / A_{j}\right) / Z x_{j} \rightarrow A /\left(A_{i} \oplus A_{j}\right) \rightarrow 0
$$

is exact. Write $A /\left(A_{i} \oplus A_{j}\right)=\bigoplus_{p} Z\left(p^{k_{p}}\right)$ so that

$$
k_{p}=\left(p \text {-height of } x_{j} \text { in } A / A_{j}\right)-\left(p \text {-height of } a_{i} \text { in } A_{i}\right) \text {. }
$$

Then $\left[\left(k_{p}\right)\right]=\sigma_{j}-\tau_{i}$. Similarly, $\left[\left(k_{p}\right)\right]=\sigma_{i}-\tau_{j}$.

Given three distinct positive integers $i, j, k$,

$\sigma_{0}-\tau_{j}=\sup \left\{\sigma_{i}-\tau_{j}, \sigma_{k}-\tau_{j}\right\}=\sup \left\{\sigma_{j}-\tau_{i}, \sigma_{j}-\tau_{k}\right\}=\sigma_{j}-\tau_{0}$.

(e) follows from (d).

Following Warfield [14], $\tau_{0}$ is called the inner type of $A$, denoted by $\operatorname{IT}(A)$, and $\sigma_{0}$ is called the outer type of $A$, denoted by $\operatorname{OT}(A)$.

LEMma 1.2. Assume that $Z x \oplus Z y \subseteq A$, a rank-2 torsion free group and that $T_{A}=\left(\tau_{1}, \tau_{2}, \ldots\right)$. Define

$$
U_{A}=\{r x+s y \mid r, s \in Z, r \geq 0, \operatorname{gcd}(r, s)=1\} .
$$

(a) For each $i \geq 1$ there is a unique $a_{i} \in U_{A} \cap A_{i}$. Moreover, $A_{i} \cap$ $(Z x \oplus Z y)=Z a_{i}$ and $\operatorname{type}_{A}\left(a_{l}\right)=\tau_{l}$.

(b) $\operatorname{OT}(A)=\left[\max \left\{h^{A}(a) \mid a \in U_{A}\right\}\right]$.

Proof. (a) is routine.

(b) Write $A /(Z x \oplus Z y)=\oplus_{p}\left[Z\left(p^{i_{p}}\right) \oplus Z\left(p^{j_{p}}\right)\right]$ with $0 \leq i_{p} \leq j_{p} \leq$ $\infty$ for each $p$. Then $\operatorname{IT}(A)=\left[\left(i_{p}\right)\right]$ and OT $(A)=\left[\left(j_{p}\right)\right]$ (Warfield [14] or Arnold [2]). If $a+(Z x \oplus Z y) \in(A /(Z x \oplus Z y))_{p}$ then order $(a+(Z x$ $\oplus Z y))=$ least $j$ with $p^{j} a=m u$ for some $u \in U_{A}$ and some $m \in Z$ with $\operatorname{gcd}(p, m)=1$. Since $j \leq h_{p}^{A}(u)$, it follows that $j_{p} \leq \max \left\{h_{p}^{A}(a) \mid a \in U\right\}$. But

$$
\begin{aligned}
A /(Z x \oplus Z y) & \supseteq\left(A_{i}+(Z x \oplus Z y)\right) /(Z x \oplus Z y) \simeq A_{i} / Z a_{t} \\
& =\bigoplus_{p} Z\left(p^{l_{p}}\right),
\end{aligned}
$$


where $l_{p}=h_{p}^{A}\left(a_{i}\right)$ (by (a)) so that $\max \left\{h_{p}^{A}(a) \mid a \in U_{A}\right\} \leq j_{p}$. Thus, OT( $\left.A\right)$ $=\left[\left(j_{p}\right)\right]=\left[\max \left\{h^{A}(a) \mid a \in U_{A}\right\}\right]$.

The following lemma reduces the problem of realizing a type sequence $T=\left(\tau_{1}, \tau_{2}, \ldots\right)$ to the case that type $(Z)=\inf \left\{\tau_{i}, \tau_{j}\right\}$ whenever $i \neq j$.

Lemma 1.3 (Schultz [11]). Let $T=\left(\tau_{1}, \tau_{2}, \ldots\right)$ and $C=\left(\sigma_{1}, \sigma_{2}, \ldots\right)$ be type sequences with $\tau_{0}=\inf \left\{\tau_{t}, \tau_{j}\right\}$ and $\sigma_{0}=\sup \left\{\sigma_{i}, \sigma_{j}\right\}$ whenever $i \neq j$. There is a rank-2 group $A$ with $T_{A}=T$ and $C_{A}=C$ iff there is a rank-2 group $B$ with $T_{B}=\left(\tau_{1}-\tau_{0}, \tau_{2}-\tau_{0}, \ldots\right), C_{B}=\left(\sigma_{1}-\tau_{0}, \sigma_{2}-\tau_{0}, \ldots\right)$, $\mathrm{IT}(B)=\operatorname{type}(Z)$, and $\mathrm{OT}(B)=\sigma_{0}-\tau_{0}$.

Proof. $(\Leftarrow)$ Let $X$ be a rank-1 group with type $(X)=\tau_{0}$ and define $A=X \otimes_{Z} B$. Then $Y$ is a pure rank-1 subgroup of $A$ iff $Y \simeq X \otimes_{Z} D$ for some pure rank-1 subgroup $D$ of $B$. Moreover, $A / Y \simeq X \otimes_{Z}(B / D)$; $\operatorname{type}(Y)=\tau_{0}+\operatorname{type}(D)$; and $\operatorname{type}(A / Y)=\tau_{0}+\operatorname{type}(B / D)$. Thus, $T_{A}=$ $T$ and $C_{A}=C$.

$(\Rightarrow)$ Choose $h_{i} \in \tau_{i}$ for $i \geq 0$ such that $h_{0} \leq h_{i}$ for each $i$. First of all, it suffices to assume that $\tau_{0}$ is idempotent: Let $X$ be a rank-1 group with type $(X)=\tau_{0}$ and $x \in X$ with $h^{X}(x)=h_{0}$. Define $A^{\prime}=\operatorname{Hom}(X, A)$. Then $\phi: A^{\prime} \rightarrow\left\{a \in A \mid h^{A}(a) \geq h_{0}\right\}$ is an isomorphism, where $\phi(f)=f(x)$. Thus $T_{A^{\prime}}=\left(\tau_{1}-\tau_{0}^{\prime}, \tau_{2}-\tau_{0}^{\prime}, \ldots\right), C_{A^{\prime}}=\left(\sigma_{1}-\tau_{0}^{\prime}, \sigma_{2}-\tau_{0}^{\prime}, \ldots\right)$, and $\operatorname{IT}\left(A^{\prime}\right)$ $=\tau_{0}-\tau_{0}^{\prime}$ where $\tau_{0}^{\prime}=\left[h_{0}^{\prime}\right], h_{0}^{\prime}(p)=0$ if $h_{0}(p)=\infty$, and $h_{0}(p)=h_{0}^{\prime}(p)$ if $h_{0}(p)<\infty$. Therefore, $\tau_{0}-\tau_{0}^{\prime}$ is idempotent.

Now assume that $\tau_{0}$ is idempotent, say $\tau_{0}=\left[h_{0}\right]$ with $h_{0}(p)=0$ or $\infty$ for each $p$. Let $F$ be a free subgroup of $A$ with $A / F$ torsion and define $B=A \cap\left(\cap\left\{F_{p} \mid h_{0}(p)=\infty\right\}\right)$. Then $B_{p}=F_{p}$ if $h_{0}(p)=\infty$ and $B_{p}=A_{p}$ if $h_{0}(p)=0$. Let $R$ be the subring of $Q$ generated by $\left\{1 / p \mid h_{0}(p)=\infty\right\}$ and define $\theta: R \otimes_{Z} B \rightarrow A$ by $\theta(r \otimes b)=r b$, noting that $R A=A$. Then $\theta$ is an epimorphism, hence an isomorphism, since $\operatorname{rank}\left(R \otimes_{Z} B\right)=$ $\operatorname{rank}(A)=2$. Finally, if $A_{i}$ is a pure rank-1 subgroup of $A$ let

$$
B_{i}=A_{i} \cap B=A_{i} \cap\left(\cap\left\{F_{p} \mid h_{0}(p)=\infty\right\}\right) .
$$

Then $B_{i}$ is a pure rank-1 subgroup of $B$ and type ${ }_{B}\left(B_{i}\right)=\tau_{l}-\tau_{0}$, since $\left(B_{i}\right)_{p}=\left(A_{i}\right)_{p}$ if $h_{0}(p)=0$ and $\bigcap\left\{\left(B_{l}\right)_{p} \mid h_{0}(p)=\infty\right\}$ is pure in $\cap\left\{F_{p} \mid h_{0}(p)=\infty\right\}$. Consequently, $T_{B}=\left(\tau_{1}-\tau_{0}, \tau_{2}-\tau_{0}, \ldots\right), C_{B}=$ $\left(\sigma_{1}-\tau_{0}, \sigma_{2}-\tau_{0}, \ldots\right)$, and $\operatorname{IT}(B)=\operatorname{type}(Z)$, as desired.

Let $T=\left(\tau_{1}, \tau_{2}, \ldots\right)$ be a type sequence with type $(Z)=\inf \left\{\tau_{i}, \tau_{j}\right\}$ whenever $i \neq j$, let $h_{i} \in \tau_{l}$ for each $i$, and let $\tau$ be a type with $h \in \tau$. Then 
$\tau$ is a snarl of $T$ if $\left\{p \mid 0<h(p)<h_{j}(p)=\infty\right.$ for some $\left.j\right\}$ is infinite. Note that this definition depends only upon $\tau$ and $T$ and not upon the choice of $h \in \tau$ and $h_{i} \in \tau_{i}$. Snarls of sets of types are defined analogously.

Suppose that $A$ is a rank-2 group with $T_{A}=\left(\tau_{1}, \tau_{2}, \ldots\right)$. By Proposition 1.1.a, $\operatorname{IT}(A)$ is the only type in $T_{A}$ that may be repeated. Following Beaumont-Pierce [3], $A$ is completely anisotropic if $\tau_{i} \neq \tau_{j}$ for each $i \neq j$. In this case, $\operatorname{IT}(A)$ appears at most one time in $T_{A}$.

TheOrem 1.4 (Dubois [1]). Let $A$ be a rank-2 group with $T_{A}=$ $\left(\tau_{1}, \tau_{2}, \ldots\right)$ and $\operatorname{IT}(A)=\operatorname{type}(Z)$. Then $T_{A}$ has an infinite subsequence $T^{\prime}$ such that no snarls of $T^{\prime}$ are in $T_{A}$.

Proof. Choose $Z x \oplus Z y \subseteq A$ with $\inf \left\{h^{A}(x), h^{A}(y)\right\}=0$. There is an indexing $u_{1}, u_{2}, \ldots$ of $U_{A}$ such that $u_{1}=x, u_{2}=y, u_{l}=r_{l} x+s_{i} y$ and $\max \left\{r_{i},\left|s_{i}\right|\right\} \leq \max \left\{r_{j},\left|s_{j}\right|\right\}$ if $i<j$. Relabel $T_{A}$ so that $\tau_{i}=\operatorname{type}_{A}\left(u_{i}\right)$. Let $h_{i}=h^{A}\left(u_{i}\right) \in \tau_{i}$ for each $i \geq 1$.

Define $K=\left\{j \mid\right.$ for each $p, h_{j}(p)<\infty$ or $h_{j}(p)=\infty$ and there is no $i<j$ with $\left.0<h_{l}(p)<h_{j}(p)=\infty\right\}$. Let $T^{\prime}$ be the subsequence of $T_{A}$ determined by $K$. Then for each $i, \tau_{i}$ is not a snarl of $T^{\prime}$ since

$$
\begin{aligned}
& \left\{p \mid 0<h_{i}(p)<h_{j}(p)=\infty, \text { for some } j \in K\right\} \\
& \subseteq\left\{p \mid 0<h_{i}(p)<h_{j}(p)=\infty, i>j \in K\right\}
\end{aligned}
$$

is finite (recalling that $\inf \left\{h_{i}(p), h_{j}(p)\right\}=0$ for almost all $p$, since $\operatorname{IT}(A)=\operatorname{type}(Z)$, and that there are only finitely many $j<i)$.

It now suffices to prove that $K$ is infinite. Let $I_{n}=\left\{i \mid \max \left\{r_{l},\left|s_{l}\right|\right\} \leq\right.$ $n\}$. If $j \in I_{n} \backslash K$ then there is some $p$ and some $i<j$ with $0<h_{i}(p)<$ $h_{j}(p)=\infty$ and $\max \left\{r_{j},\left|s_{j}\right|\right\} \leq n$. Now $r_{i} u_{j}=r_{j} u_{i}-\left(r_{j} s_{l}-r_{i} s_{j}\right) y$ and $s_{\imath} u_{j}=s_{j} u_{i}+\left(r_{j} s_{i}-r_{i} s_{j}\right) x$. Since $\inf \left\{h_{p}^{A}(x), h_{p}^{A}(y)\right\}=0, p$ divides $r_{j} s_{l}-$ $r_{i} s_{j}$. Furthermore, $\left|r_{j} s_{i}-r_{i} s_{j}\right| \leq 2 n^{2}$. Thus, $\left|I_{n} \backslash K\right| \leq \pi\left(2 n^{2}\right)$, the number of primes $\leq 2 n^{2}$, since for each $p$ there is at most one $j$ with $h_{j}(p)=\infty$. It follows that

$$
\left|I_{n} \cap K\right|=\left|I_{n}\right|-\left|I_{n} \backslash K\right| \geq\left|I_{n}\right|-\pi\left(2 n^{2}\right) .
$$

Now apply Lemma 0.1.e to see that $K$ is infinite.

EXAMPLE 1.5. Let $T=\left(\tau_{1}, \tau_{2}, \ldots\right)$ be given by $\tau_{i}=\left[h_{l}\right]$ where $h_{1}=$ $(1,1,1, \ldots) ; h_{2}=(\infty, 0,0, \ldots) ; h_{3}=(0, \infty, 0, \ldots), h_{4}=(0,0, \infty, \ldots), \ldots$.

(a) There is no rank-2 group $A$ with $T_{A}=T$.

(b) There is no rank-2 group $A$ with $\operatorname{typeset}(A)=\left\{\tau_{l} \mid i \geq 1\right\}$.

(c) There is no rank-2 completely anisotropic group $A$ with $\operatorname{typeset}(A)$ $=\left\{\tau_{l} \mid i \geq 0\right\}$ where $\tau_{0}=\operatorname{type}(Z)=\inf \left\{\tau_{l}, \tau_{j}\right\}$ for $i \neq j$. 
Proof. (a) Note that $\tau_{1}$ is a snarl of every infinite subsequence of $T$ and apply Theorem 1.4.

(b) If there is a rank-2 group with typeset $(A)=\left\{\tau_{i} \mid i \geq 1\right\}$ then $\operatorname{IT}(A)=\operatorname{type}(Z) \notin \operatorname{typeset}(A)$. Thus $T_{A}=\left(\tau_{1}, \tau_{2}, \ldots\right)$ since $\operatorname{IT}(A)$ is the only type that can be repeated and $\operatorname{IT}(A)$ does not appear in $T_{A}$. But this contradicts (a).

(c) For such an $A, T_{A}=\left(\tau_{0}, \tau_{1}, \tau_{2}, \ldots\right)$ since $A$ is assumed to be completely anisotropic. Once again, $\tau_{1}$ is a snarl of every infinite subsequence of $T_{A}$ contradicting Theorem 1.4.

EXAmple 1.6. Let $S_{1}=\left\{\tau_{l} \mid i \geq 1\right\}$ be as defined in Example 1.5, $\sigma_{0}=\operatorname{type}(Q)$, and $S_{2}=\left\{\sigma_{0}-\tau_{i} \mid i \geq 1\right\}$. Then there is no rank-2 group $A$ with $\operatorname{typeset}(A)=S_{1}$ and $\operatorname{cotypeset}(A)=S_{2}$ by Example 1.5. On the other hand, $S_{1}$ and $S_{2}$ satisfy the hypotheses of Theorem 1, Schultz [11]. Thus Schultz's main theorem is incorrect as stated.

2. Realization of type sequences and typesets. In this section the following notation is consistently employed: $T=\left(\tau_{1}, \tau_{2}, \ldots\right)$ is a type sequence with $\inf \left\{\tau_{l}, \tau_{j}\right\}=\operatorname{type}(Z)$ if $i \neq j ; h_{l} \in \tau_{i}$ for all $i ; a_{l}=r_{i} x+s_{i} y$ is a denumeration of $U=\{r x+s y \in Z x \oplus Z y \mid r, s \in Z, r \geq 0$, and $\operatorname{gcd}(r, s)=1\} ; \operatorname{det}(i, j)=r_{l} s_{j}-s_{l} r_{j} ;$ and $\operatorname{det}_{p}(i, j)=h_{p}^{Z}\left(r_{l} s_{j}-s_{i} r_{j}\right)$.

The type sequence $T$ is admissible if there is an indexing of $U$ so that for each $i$ there is an $N>0$ such that $h_{n}(p)=\infty$ for some $n>N$ implies $\operatorname{det}_{p}(i, n)=h_{i}(p)$.

Since for each $p$ there is at most one $n$ with $h_{n}(p)=\infty$, the admissibility of $T$ does not depend on the choice of $h_{l} \in \tau_{l}$ or the ordering of $T$.

LEMMA 2.1. Given $h_{i}^{\prime} \in \tau_{l}$ for each $i$, there exists $h_{i} \in \tau_{l}$ for each $i$ such that $h_{i} \leq h_{l}^{\prime}$ and (a) If $j<k$ and $h_{k}(p)<\infty$ then $\min \left\{h,(p), h_{k}(p)\right\}=0$; and (b) If $h_{k}(p)<\infty$ and $\operatorname{det}_{p}(i, k)>0$ for some $i<k$ then $h_{k}(p)=0$.

Proof. Assume $h_{1}, \ldots, h_{n-1}$ have been chosen such that $h_{t} \in \tau_{l}, h_{i} \leq h_{l}^{\prime}$ and (a) and (b) are satisfied for $i, j, k<n$. Define $h_{n}(p)=0$ if $h_{n}^{\prime}(p)<\infty$ and either $0<h_{i}(p)$ for some $i<n$ or $\operatorname{det}_{p}(i, n)>0$ for some $i<n$; and define $h_{n}(p)=h_{n}^{\prime}(p)$ otherwise.

Note that there are only finitely many $i<n$ and only a finite number of primes can divide $\operatorname{det}(i, n)$ if $i \neq n$. Furthermore, $h_{n}^{\prime}(p)>0$ and $h_{l}(p)>0$ for some $i<n$ can happen for only finitely many $p$ since $\inf \left\{\tau_{n}, \tau_{i}\right\}=\operatorname{type}(Z)$. Thus $h_{n}(p)=h_{n}^{\prime}(p)$ in case $h_{n}^{\prime}(p)=\infty$ and for almost all $p$, and $h_{n}(p) \leq h_{n}^{\prime}(p)$. Therefore $h_{n} \in \tau_{n}$ and $h_{1}, \ldots, h_{n}$ satisfy (1) and (2). The proof is now complete by induction on $n$. 
Lemma 2.2. Suppose that $h_{i} \in \tau_{i}$ for each $i$. Define $A$ to be the subgroup of $Q x \oplus Q y$ generated by $\left\{a_{i} / p^{j} \mid p\right.$ is a prime, $\left.0 \leq j \leq h_{l}(p), i=1,2, \ldots\right\}$. Then $\mathrm{OT}(A)=[h]$ where $h=\max \left\{h_{i} \mid i \geq 1\right\}$.

Proof. By Proposition 1.1(a),

$$
\mathrm{OT}(A)=\sup \left\{\operatorname{type}\left(A / A_{1}\right), \operatorname{type}\left(A / A_{2}\right)\right\},
$$

where $A_{1}$ and $A_{2}$ are the pure rank-1 subgroups of $A$ generated by $x$ and $y$, respectively. Since

$\operatorname{type}\left(A / A_{1}\right)=\operatorname{type}\left\langle s_{i} / p^{j} \in Q\right| p$ is a prime, $\left.0 \leq j \leq h_{i}(p), i=1,2, \ldots\right\rangle$, $\operatorname{type}\left(A / A_{2}\right)=\operatorname{type}\left\langle r_{l} / p^{j} \in Q\right| p$ is a prime, $\left.0 \leq j \leq h_{l}(p)\right\rangle$, and $\operatorname{gcd}\left(r_{i}, s_{l}\right)=1$ for each $i$, the result follows.

LEMmA 2.3. Let $p$ be a prime, and $Z x \oplus Z y \subseteq A \subseteq Q x \oplus Q y$ with $h_{p}^{A}\left(a_{l}\right)=0$ for each $i, B=\left\langle A \cup\left\{a_{k} / p^{j} \mid j \leq e\right\}\right\rangle$ for some $k>0$, and $0<e \leq \infty$. Then for each $i, h_{p}^{B}\left(a_{i}\right)=\min \left\{e, \operatorname{det}_{p}(i, k)\right\}$.

Proof. Fix $i>0$ and assume that $\operatorname{gcd}\left(p, s_{k}\right)=1$. We first show that $h_{p}^{B}(x)=0$. Suppose that $x / p=a+c a_{k} / p^{l}$ for some $a \in A, c \in Z, 0 \leq l$ $\leq e, l<\infty$. Then $x=p a+c a_{k} / p^{l-1}$ so $c a_{k} / p^{l-1} \in A$. Thus we may assume that $c / p^{l-1}=c^{\prime} \in Z$ with $\operatorname{gcd}\left(c^{\prime}, p\right)=1$. By Lemma 1.2, $a=$ $(m / n) a_{1}$ for some $i \neq k, \operatorname{gcd}(m, n)=1$. Equating coefficients of $y$ gives $0=(p m / n) s_{i}+c^{\prime} s_{k}$. Since $\operatorname{gcd}\left(c^{\prime} s_{k}, p\right)=1, p$ divides $n$. This contradicts $h_{p}^{A}\left(a_{l}\right)=0$.

The lemma now follows, in this case, from the equation $s_{k} a_{i}-s_{i} a_{k}=$ $\operatorname{det}(i, k) x$. Indeed, since $h_{p}^{B}(x)=0$ and $\operatorname{gcd}\left(s_{k}, p\right)=1$,

$$
h_{p}^{B}\left(a_{l}\right) \geq \min \left\{e, \operatorname{det}_{p}(i, k)\right\} \text {. }
$$

On the other hand, if $p^{l}$ divides $a_{l}$ in $B$, then $l \leq e$ by the construction of $B$, so $p^{l}$ divides $a_{k}$ in $B$. Since $h_{p}^{B}(x)=0$, the equation implies $p^{l}$ divides $\operatorname{det}(i, k)$. Hence, $h_{p}^{B}\left(a_{i}\right) \leq \min \left\{e, \operatorname{det}_{p}(i, k)\right\}$ as desired.

A similar argument shows that if $\operatorname{gcd}\left(p, r_{k}\right)=1$, then $h_{p}^{B}(y)=0$ and again $h_{p}^{B}\left(a_{i}\right)=\min \left\{e, \operatorname{det}_{p}(i, k)\right\}$. Since $\operatorname{gcd}\left(r_{k}, s_{k}\right)=1$, the proof is complete.

Lemma 2.4. Let $p$ be a prime, $Z x \oplus Z y \subseteq A \subseteq Q x \oplus Q y$ with $h_{p}^{A}\left(a_{l}\right)$ $=0$ for each $i, \alpha$ an irrational $p$-adic integer and $0<t \leq \infty$. Define

$$
B=\left\langle A \cup\left\{a_{l} / p^{j} \mid p^{j} \text { divides } r_{l}-\alpha s_{i} \text { and } j \leq t, 1 \leq i<\infty\right\}\right\rangle .
$$


Then

$$
h_{p}^{B}\left(a_{l}\right)=\max \left\{j \mid p^{\jmath} \text { divides } r_{t}-\alpha s_{l} \text { and } j \leq t\right\}<\infty
$$

for each $i$.

Proof. Given $i$, let $m=\max \left\{j \mid p^{\prime}\right.$ divides $r_{l}-\alpha s_{i}$ and $\left.j \leq t\right\}$. Note that if $p$ divides $r_{l}-\alpha s_{l}$ then $\operatorname{gcd}\left(s_{l}, p\right)=1$ since $\operatorname{gcd}\left(s_{i}, r_{i}\right)=1$. Clearly, $h_{p}^{B}\left(a_{l}\right) \geq m$ and $m<\infty$. It therefore suffices to show $h_{p}^{B}\left(a_{l}\right)$ is not greater than $m$. Suppose $\left(1 / p^{m+1}\right) a_{i} \in B$. Then $1 / p^{m+1} a_{l}=a+\sum_{k=1}^{l} c_{k} a_{k} / p^{e(k)}$, where $a \in A, c_{k} \in Z, p^{e(k)}$ divides $r_{k}-\alpha s_{k}$ and $e(k) \leq t$. Choose $1 \leq j \leq l$ so that $e(j)$ is maximal among the $e(k)$. Note that

$$
s_{j} a_{k}=s_{k} a_{j}+s_{k}\left(r_{j}-\alpha s_{j}\right) x+s_{j}\left(\alpha s_{k}-r_{k}\right) x .
$$

Since $e(j)$ is maximal and $\operatorname{gcd}\left(s_{j}, p\right)=1$, this equation implies that each $\left(c_{k} / p^{e(k)}\right) a_{k}$ may be replaced by an expression of the form $b_{k}+$ $\left(c_{k}^{\prime} / p^{e(\jmath)}\right) a_{j}$ where $b_{k} \in A$ and $c_{k}^{\prime} \in Z$. Thus we may write $\left(1 / p^{m+1}\right) a_{t}$ $=a+\left(c / p^{e}\right) a$, where $a \in A, c \in Z, p^{e}$ divides $r_{\text {, }}-\alpha s$, and $e \leq t$. This shows that $\left(1 / p^{m+1}\right) a_{t}$ is in fact an element of the group $A^{\prime}=A \cup$ $\left\{a_{j} / p^{i} \mid i \leq e\right\}$. By Lemma 2.3, $m+1 \leq \min \left\{e, \operatorname{det}_{p}(i, j)\right\}$. In particular, $\operatorname{det}_{p}(i, j) \geq m+1$. Therefore, $p^{m+1} \operatorname{divides}\left(r_{i} s_{,}-s_{l} r_{j}\right)$. Since $e \geq m+1$, $p^{m+1}$ divides $\left(r_{,}-\alpha s_{l}\right) s_{l}$; thus $p^{m+1}$ divides $r_{l} s_{l}-\alpha s_{\text {J }} s_{l}$ and $p$ divides $s_{\text {, }}$. However $p$ divides $r_{j}-\alpha s_{\text {, }}$, so $p$ divides $r_{J}$ contradicting $\operatorname{gcd}\left(r_{j}, s_{J}\right)$ $=1$. Thus $h_{p}^{B}\left(a_{l}\right)$ is not greater than $m$.

The next theorem is stated in Dubois [6].

THEOREM 2.5. There is a rank-2 group $A$ with $T_{A} \approx T$ if and only if $T$ is admissible.

Proof. $(\Rightarrow)$ Assume that $Z x \oplus Z y \subseteq A \subseteq Q x \oplus Q y$ and let $a_{1}=x, a_{2}$ $=y, a_{3}, \ldots$ be an indexing of $U$ such that type ${ }_{A}\left(a_{l}\right)=\tau_{l}$ for each $i$. Define $h_{l}=h^{A}\left(a_{i}\right)$ for each $i$, let

$$
N=\max \left\{j \mid h_{j}(p)=\infty, \inf \left\{h_{1}(p), h_{2}(p)\right\}>0\right\},
$$

and let $N=1$ if no such $j$ exists. Given $i$ and $n>N$ with $h_{n}(p)=\infty$, then $h_{l}(p)=\operatorname{det}_{p}(n, i)$ since $s_{l} a_{n}-s_{n} a_{l}=\operatorname{det}(n, i) x$ and $r_{n} a_{l}-r_{l} a_{n}=$ $\operatorname{det}(n, i) y$. Note that for this choice of $h_{i} \in \tau_{l}, N$ does not depend on $i$.

$(\Leftarrow)$ If $T$ is admissible, choose $h_{l} \in \tau_{l}$ satisfying (a) and (b) of Lemma 2.1. Next define $h_{l}^{\prime} \leq h_{l}$ by setting $h_{l}^{\prime}(p)=0$ if $h_{k}(p)=\infty$ for some $k \neq i$ and $h_{i}^{\prime}(p)=h_{i}(p)$ otherwise. Note that $h_{i}^{\prime}$ need not be in $\tau_{l}$. Given $p$, this 
implies (along with (a)) that $h_{l}^{\prime}(p)>0$ for at most one $i$. Let

$$
A(p)=\left\langle(Z x \oplus Z y) \cup\left\{a_{l} / p^{j} \mid 0 \leq j \leq e(i)\right\}\right\rangle
$$

if $e(i)=h_{i}^{\prime}(p)>0$ for some $i$, and let $A(p)=Z x \oplus Z y$ otherwise. Define $A=\Sigma_{p} A(p)$. Note that $h_{p}^{A}\left(a_{i}\right)=h_{p}^{A(p)}\left(a_{i}\right)$ for all $i$ and $p$, so we can apply Lemma 2.3 to show type ${ }_{A}\left(a_{i}\right)=\tau_{l}$ for all $i$ as follows:

Let $P_{1}=\left\{p \mid h_{i}^{\prime}(p)=0\right.$ for each $\left.i\right\}$. Then for each $p \in P_{1}, h_{p}^{A}\left(a_{l}\right)=0$ $=h_{l}(p)$ for each $i$.

Let $P_{2}=\left\{p \mid h_{k}^{\prime}(p)=\infty\right.$ for some $\left.k=k(p)\right\}$. If $p \in P_{2}$ then $h_{p}^{A}\left(a_{i}\right)$ $=\operatorname{det}_{p}(i, k(p))$ by Lemma 2.3. By the admissibility condition, $h_{p}^{A}\left(a_{t}\right)=$ $h_{l}(p)$ for almost all $p \in P_{2}$.

Let $P_{3}=\left\{p \mid 0<h_{k}^{\prime}(p)=h_{k}(p)<\infty\right.$ for some (unique) $\left.k=k(p)\right\}$. If $p \in P_{3}$, then

$$
h_{p}^{A}\left(a_{i}\right)=\min \left\{h_{k(p)}(p), \operatorname{det}_{p}(i, k(p))\right\} .
$$

If $i=k(p)$ then $h_{p}^{A}\left(a_{l}\right)=h_{k(p)}(p)$. On the other hand, if $i \neq k(p)$ and $\operatorname{det}_{p}(i, k(p))>0$ then $k(p)<i$ by condition (b) on the $h_{i}$ 's. This implies $h_{p}^{A}\left(a_{l}\right)=0=h_{l}(p)$ except for a finite number of $p$. Thus $h_{p}^{A}\left(a_{i}\right)=h_{l}(p)$ for almost all $p \in P_{3}$. Consequently, type ${ }_{A}\left(a_{l}\right)=\tau_{i}$ for each $i$ as desired.

THEOREM 2.6. Given a rank-2 group $A$ and a type $\sigma \geq \mathrm{OT}(A)$, there is a rank-2 group $B$ with $\mathrm{OT}(B)=\sigma$ and $T_{A}=T_{B}$.

Proof. Assume that $Z x \oplus Z y \subseteq A \subseteq Q x \oplus Q y$. Choose $h \in \sigma$ and $h_{l} \in \operatorname{type}_{A}\left(a_{i}\right)$ such that $h \geq h_{l}$ for each $i$, and such that the $h_{l}$ 's satisfy (a) and (b) of Lemma 2.1. Note that $h \geq \max \left\{h_{l} \mid i \geq 1\right\}$ and that $h(p)=\infty$ for all $p$ in $P_{\infty}=\left\{p \mid h_{k}(p)=\infty\right.$ for some $\left.k\right\}$. In view of Theorem 2.5, it suffices to assume that $A=\Sigma_{p} B(p)$, where

$$
B(p)=\left\langle Z x \oplus Z y \cup\left\{a_{l} / p^{\prime} \mid 0 \leq j \leq h_{l}^{\prime}(p), i=1,2, \ldots\right\}\right\rangle,
$$

$h_{l}^{\prime}(p)=0$ if $p \in P_{\infty}$ and $h_{i}(p)<\infty, h_{l}^{\prime}(p)=h_{l}(p)$ otherwise. Thus, $\min \left\{h_{i}^{\prime}(p), h_{j}^{\prime}(p)\right\}=0$ for each $p$ and $i \neq j$. We will construct $B$ using Lemma 2.4. This involves choosing an index $k=k(p)$ and a $p$-adic integer $\alpha=\alpha(p)$ for an appropriate collection of primes $p$.

First consider $P_{1}=\left\{p \mid h(p)>0\right.$ and $h_{i}(p)=0$ for all $\left.i\right\}$. Write $P_{1}=\{q(1), q(2), \ldots\}$ with $q(i)<q(i+1)$. For each $p=q(t) \in P_{1}$, let $k(p)=t$, and let $\alpha(p)$ be an irrational $p$-adic integer such that $p$ does not divide $r_{t}-\alpha(p) s_{l}$ for $i \leq t$.

Next consider $P_{2}=\left\{p \mid 0<h_{k}^{\prime}(p)<h(p) \leq \infty\right.$ for some (unique) $k=k(p)\}$. Assume $\operatorname{gcd}\left(s_{k}, p\right)=1$ and choose an irrational $p$-adic integer 
$\alpha=\alpha(p)$ with $p$-height $\left(r_{k}-\alpha s_{k}\right)=h_{k}^{\prime}(p)$. (If $p$ divides $s_{k}$, then $\operatorname{gcd}\left(r_{k}, p\right)=1$ and the roles of $s$ and $r$ may be reversed in the proof. For example, $\alpha$ would be chosen so that $p$-height $\left(\alpha r_{k}-s_{k}\right)=h_{k}^{\prime}(p)$.)

Denote $P_{3}=P_{1} \cup P_{2}$. For $p \in P_{3}$, let $k=k(p), \alpha=\alpha(p)$, and let

$$
A(p)=\left\langle Z x \oplus Z y \cup\left\{a_{i} / p^{\prime} \mid p^{j} \text { divides } r_{i}-\alpha s_{i} \text { and } j \leq h(p)\right\}\right\rangle .
$$

By Lemma 2.4, $h_{p}^{A(p)}\left(a_{i}\right)<\infty$ for each $i$. Moreover, if $p \in P_{2}$ and $h_{p}^{A(p)}\left(a_{l}\right)>0$, then $p$ divides $r_{i}-\alpha s_{l}$, and hence

(*) $\quad p$ divides $s_{k}\left(r_{i}-\alpha s_{i}\right)-s_{l}\left(r_{k}-\alpha s_{k}\right)=s_{k} r_{i}-s_{i} r_{k}=\operatorname{det}(i, k)$.

Define $B=\Sigma\left\{A(p) \mid p \in P_{3}\right\}+\Sigma\left\{B(p) \mid p \notin P_{3}\right\}$. Then $h_{p}^{B}\left(a_{i}\right)=$ $h_{p}^{A(p)}\left(a_{i}\right)$ for each $i$ and $p \in P_{3}$. By Lemma 2.2, OT $(B)$ and $\sigma$ agree on $P_{3}$, and therefore $\operatorname{OT}(B)=\sigma$. To see that $\operatorname{type}_{A}\left(a_{l}\right)=\operatorname{type}_{B}\left(a_{i}\right)$, first note that if $p=q(t)$ is an element of $P_{1}$, then for $i \leq t, h_{p}^{B}\left(a_{l}\right)=0$ by Lemma 2.4 and the choice of $\alpha(p)$. Thus type ${ }_{A}\left(a_{i}\right)$ and type ${ }_{B}\left(a_{l}\right)$ agree on $P_{1}$. Next let $p \in P_{2}$. If $i=k(p)$, then

$$
\begin{aligned}
h_{p}^{B}\left(a_{i}\right) & =\max \left\{j \mid p^{j} \text { divides } r_{l}-\alpha(p) s_{i} \text { and } j \leq h(p)\right\} \\
& =h_{i}(p)=h_{l}^{\prime}(p)
\end{aligned}
$$

by Lemma 2.4 (since $h_{k(p)}(p)<h(p)$ ). Moreover, as in the proof of Theorem 2.5, $h_{p}^{A}\left(a_{i}\right)=h_{l}^{\prime}(p)$, so that $h_{p}^{B}\left(a_{i}\right)=h_{p}^{A}\left(a_{i}\right)$ in this case. On the other hand, if $i \neq k(p)$ and $h_{p}^{B}\left(a_{i}\right)>0$, then $p$ divides $\operatorname{det}(i, k(p))$ as shown above (*). By condition (b), this happens only if $k(p)>i$, since $0<h_{k(p)}(p)<\infty$. Thus, $i \neq k(p)$ and $h_{p}^{B}\left(a_{l}\right)>0$ can happen for at most finitely many $p \in P_{2}$, and $h_{p}^{A}\left(a_{i}\right)=h_{p}^{B}\left(a_{l}\right)$ for almost all $p \in P_{2}$. It follows that $\operatorname{type}_{A}\left(a_{i}\right)=\operatorname{type}_{B}\left(a_{i}\right)$.

LEMma 2.7 (Dubois [5]). Let $T^{\prime}=\left(\tau_{1}^{\prime}, \tau_{2}^{\prime}, \ldots\right)$ be a type sequence with $\operatorname{type}(Z)=\inf \left\{\tau_{l}^{\prime}, \tau_{j}^{\prime}\right\}$ whenever $i \neq j$. Assume that $T^{\prime}$ has an infinite subsequence $T_{0}^{\prime}$ with no snarls of $T_{0}^{\prime}$ in $T^{\prime}$. Then there is a type sequence $T=\left(\tau_{1}, \tau_{2}, \ldots\right)$ and $h_{i} \in \tau_{i}$ for each $i$ such that $T \approx T^{\prime}$ and

(a) If $p_{i}$ is the ith prime, then $h_{j}\left(p_{i}\right)=0$ whenever $j \geq 2 i$.

(b) If $K=\left\{k \mid\right.$ for each $p$ either $h_{k}(p)<\infty$ or else $h_{k}(p)=\infty$ and there is no $j<k$ with $\left.0<h_{j}(p)<h_{k}(p)=\infty\right\}$ then $\left\{k \mid \tau_{k}^{\prime} \in T_{0}^{\prime}\right\} \subseteq K$ so that $K$ is infinite.

Proof. (a) Let $T_{1}$ be the subsequence of types in $T^{\prime}$ with an infinity at some $p$ and let $T_{2}$ be the complement of $T_{1}$ in $T^{\prime}$. Order the elements of $T_{1}$ 
so that if $\tau_{i}^{\prime}=\left[h_{l}^{\prime}\right], \tau_{j}^{\prime}=\left[h_{j}^{\prime}\right]$ are elements of $T_{1}$ and $\min \left\{p \mid h_{l}^{\prime}(p)=\infty\right\}$ $<\min \left\{p \mid h_{j}^{\prime}(p)=\infty\right\}$ then $\tau_{l}^{\prime} \leq \tau_{j}^{\prime}$. Let $T$ be the type sequence defined by:

$\tau_{2 l}=i$ th element in $T_{2}$ (if it exists)

$\tau_{2 \imath-1}=i$ th element in $T_{1}$ (if it exists)

If either $T_{1}$ or $T_{2}$ is finite, use the elements of the infinite sequence when the elements of the finite sequence are exhausted and if both are finite, use type $(Z)$. By Lemma 2.1.a there is $h_{i} \in \tau_{l}$ for each $i$ so that if $h_{i}(p)>0$ and $j>i$, then $h_{j}(p)=0$ or $\infty$. It follows that (a) is satisfied by $T \approx T^{\prime}$.

(b) It suffices to assume that for each $j,\left\{p \mid 0<h_{j}(p)<h_{k}(p)=\infty\right.$

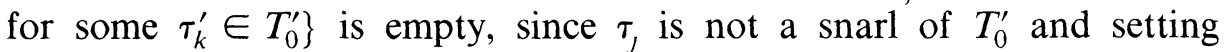
$h_{j}(p)=0$ for only finitely many $p$ with $h_{j}(p)<\infty$ does not change the type of $h_{\text {, }}$. Consequently, $\left\{k \mid \tau_{k}^{\prime} \in T_{0}^{\prime}\right\} \subseteq K$ and $K$ is infinite. Note that (a) is still satisfied.

Lemma 2.8. Suppose that $\left(r_{1}, s_{1}\right),\left(r_{2}, s_{2}\right), \ldots,\left(r_{n}, s_{n}\right)$ are distinct elements of $U ; q_{1}, q_{2}, \ldots, q_{m}$ distinct primes with each $q_{J}>n$ and $\operatorname{det}_{q}(i, l)=0$ for $q=q_{J}, 1 \leq i \neq l \leq n$, and $1 \leq j \leq m ; e_{1}, e_{2}, \ldots, e_{m}$ non-negative integers; and $\left\{i_{1}, i_{2}, \ldots, i_{m}\right\} \subseteq\{1,2, \ldots, n\}$. Then there are infinitely many $(r, s)$ in $U$ such that

$$
\begin{aligned}
& \text { if } 1 \leq j \leq m \text { and } q=q_{J} \text { then } h_{q}^{Z}\left(r s_{l}-r_{i} s\right)=0 \text { for } 1 \leq i \\
& \neq i_{j} \leq n \text { and } h_{q}^{Z}\left(r s_{i}-r_{i} s\right)=e_{j} \text { for } i=i_{1} .
\end{aligned}
$$

Proof. The proof is by induction on $m$. Suppose that $m=1$. Let $i=i_{1}, q=q_{1}$ and $e=e_{1}$. First assume that $e=0$. For each $i$ with $1 \leq i \leq n$, there is at most one $t$ with $1 \leq t \leq q$ such that $q$ divides $t s_{i}-r_{l}$. Indeed, if $q$ divides $t s_{i}-r_{l}$ and $q$ divides $t^{\prime} s_{l}-r_{i}$, then $q$ divides $\left(t-t^{\prime}\right) s_{l}$ so that $t=t^{\prime}$ or $q$ divides $s_{l}$. The latter case is impossible since $\operatorname{gcd}\left(r_{l}, s_{l}\right)$ $=1$. Since $n<q$, there must exist some $1 \leq t \leq q$, such that $h_{q}^{Z}\left(t s_{t}-r_{l}\right)$ $=0$ for each $i$. In this case $(r, s)=(t, 1) \in U$ satisfies $(*)$. Next assume that $e>0$. Choose $(r, s) \in U$ with $r s_{i}-r_{i} s=q^{e}$. Then $h_{q}^{Z}\left(r s_{l}-r_{l} s\right)=0$ whenever $1 \leq i \neq l \leq n$, otherwise $\operatorname{det}_{q}(i, l)=h_{q}^{Z}\left(r_{l} s_{l}-r_{l} s_{l}\right)>0$, which is impossible. Hence $(r, s)$ satisfies $(*)$. Given $(r, s) \in U$ satisfying $(*)$ let $x^{\prime}=r+a q^{e+1}, y^{\prime}=s+b q^{e+1}, d=\operatorname{gcd}\left(x^{\prime}, y^{\prime}\right), x=x^{\prime} / d$ and $y=y^{\prime} / d$. Then $(x, y) \in U$ and there are infinitely many such $(x, y)$ which satisfy $(*)$.

Now assume inductively that $\left(r^{\prime}, s^{\prime}\right) \in U$ satisfies (*) for $1 \leq j<m$. Let $i=i_{m}, q=q_{m}, e=e_{m}$, and let $\pi$ be the product of $\left\{q_{j}^{n(j)} \mid 1 \leq j \leq m\right.$ $\left.-1, n(j)=e_{j}+1\right\}$. Assume that $e=0$. Since $n<q$ there is $1 \leq t \leq q$ such that $h_{q}^{Z}\left(\left(r^{\prime}+t \pi\right) s_{l}-s^{\prime} r_{l}\right)=0$ for each $1 \leq l \leq n$ (as above). Let 
$r=\left(r^{\prime}+t \pi\right) / d, s=s^{\prime} / d$, where $d=\operatorname{gcd}\left(r^{\prime}+t \pi, s^{\prime}\right)$. Then $(r, s) \in U$ satisfies $(*)$ since if $p=q_{j} \neq q$ then

$$
h_{p}^{Z}\left(\left(r^{\prime}+t \pi\right) s_{l}-s^{\prime} r_{l}\right)=h_{p}^{Z}\left(r^{\prime} s_{l}-s^{\prime} r_{l}\right) \quad \text { for each } 1 \leq l \leq n .
$$

Next assume that $e \neq 0$. Choose $a, b \in Z$ with $a r_{l}+b s_{l}=1$. By the Chinese Remainder Theorem there is $x \geq 0, y$ in $Z$ with $x \equiv r^{\prime}(\bmod \pi)$, $x \equiv b q e+r_{i}\left(\bmod q^{e+1}\right)$ and $y \equiv s^{\prime}(\bmod \pi), y \equiv-a q^{e}+s_{i}\left(\bmod q^{e+1}\right)$. Then $h_{q}^{Z}\left(x s_{i}-r_{i} y\right)=e, h_{q}^{Z}\left(x s_{l}-r_{l} y\right)=0$ if $l \neq i$, and if $p=q_{j} \neq q$ then $h_{p}^{Z}\left(x s_{l}-r_{l} y\right)=h_{p}^{Z}\left(r^{\prime} s_{l}-r_{l} s^{\prime}\right)$. Consequently, $(r, s) \in U$ satisfies $(*)$, where $r=x / d, s=y / d$ and $d=\operatorname{gcd}(x, y)$.

If $x^{\prime}=r+u \pi q^{e+1}, y^{\prime}=s+v \pi q^{e+1}, d=\operatorname{gcd}\left(x^{\prime}, y^{\prime}\right), r^{\prime}=x^{\prime} / d$, and $s^{\prime}=y^{\prime} / d$ then $\left(r^{\prime}, s^{\prime}\right) \in U$ and there are infinitely many such $\left(r^{\prime}, s^{\prime}\right)$ satisfying $(*)$. By induction on $m$, the proof is complete.

A type $\tau$ is very large if $h \in \tau$ implies that $\{p \mid h(p)=\infty\}$ is infinite.

THEOREM 2.9. Suppose that $T=\left(\tau_{1}, \tau_{2}, \ldots\right)$ is a type sequence having an infinite subsequence $T_{0}$ with no snarls in $T$ and that $\operatorname{type}(Z)=\inf \left\{\tau_{i}, \tau_{j}\right\}$ whenever $i \neq j$.

(a) There is a rank-2 group $A$ with $\operatorname{IT}(A)=\operatorname{type}(Z)$ and $T_{A}=$ $\left(\tau_{1}^{\prime}, \tau_{2}^{\prime}, \ldots\right)$, where $\tau_{i}^{\prime} \geq \tau_{i}$ for each $i$ and if $h_{l}^{\prime} \in \tau_{i}^{\prime}, h_{i} \in \tau_{i}$ then $h_{l}^{\prime}(p)=\infty$ iff $h_{t}(p)=\infty$.

(b) If $\left\{\tau_{j} \mid \tau_{j}\right.$ very large $\}$ has no snarls in $T$ then $A$ may be chosen with $T_{A} \approx T$.

Proof. (a) By Lemmas 2.7 and 2.1 it suffices to assume that there is $h_{l} \in \tau_{i}$ for each $i$ such that if $j<k$ then $\inf \left\{h_{j}(p), h_{k}(p)\right\}=0$ unless $h_{k}(p)=\infty ;\left\{k \mid \tau_{k} \in T_{0}\right\} \subseteq K=\left\{k \mid\right.$ for each $p$ either $h_{k}(p)<\infty$ or else $h_{k}(p)=\infty$ and there is no $j$ with $\left.0<h_{j}(p)<h_{k}(p)=\infty\right\}$; and $h_{j}\left(p_{l}\right)$ $=0$ whenever $j \geq 2 i$ and $p_{l}$ is the $i$ th prime.

To construct an indexing of $U$, via Lemma 2.8 , let $u_{1}=(1,0)$ and $u_{2}=(0,1)$.

If $k \geq 3$ and $k \in K$ choose $u_{k}=\left(r_{k}, s_{k}\right) \in U$ with $\max \left\{r_{k},\left|s_{k}\right|\right\}$ minimal among the elements of $U$ not already chosen.

If $k \geq 3, k \notin K$, and $\tau_{k}$ is not very large let $\left\{q_{1}, q_{2}, \ldots, q_{m}\right\}=\{p \mid$ $\left.h_{k}(p)=\infty\right\} ; e_{j}=h_{l}\left(q_{j}\right)$ and $i_{J}=l$ if $0<h_{l}\left(q_{j}\right) ; e_{j}=0$ and $i_{J}=l$ for some arbitrary $l<k$ if $h_{i}\left(q_{j}\right)=0$ for all $1 \leq i<k$; and let $n_{k}$ be the largest integer less than $k$ such that $q_{J}>n_{k}$ and $\operatorname{det}_{q_{j}}(i, l)=0$ whenever $1 \leq i \neq l \leq n_{k}$ and $1 \leq j \leq m$. By Lemma 2.8, there is $u_{k^{-}}=\left(r_{k}, s_{k}\right) \in U$, not already chosen, such that $h_{l}(p)=\operatorname{det}_{p}(k, i)$ whenever $p \in$ $\left\{q_{1}, q_{2}, \ldots, q_{m}\right\}$ and $1 \leq i \leq n_{k}$. 
If $k \geq 3, k \notin K$, and $\tau_{k}$ is very large let $\left\{q_{1}, q_{2}, \ldots, q_{m}\right\}=\{p \mid 0<$ $h_{j}(p)<h_{k}(p)=\infty$ for some $\left.j<k\right\} ; e_{j}=h_{l}\left(q_{j}\right)$ and $i_{j}=l$ if $0<h_{l}\left(q_{j}\right)$; and let $n_{k}$ be the largest integer less than $k$ such that $q_{j}>n_{k}$ and $\operatorname{det}_{q_{j}}(i, l)=0$ whenever $1 \leq i \neq l \leq n_{k}$ and $1 \leq j \leq m$. By Lemma 2.8 there is $u_{k}=\left(r_{k}, s_{k}\right) \in U$, not already chosen, such that $h_{i}(p)=$ $\operatorname{det}_{p}(k, i)$ whenever $p \in\left\{q_{1}, q_{2}, \ldots, q_{m}\right\}$ and $1 \leq i \leq n_{k}$.

Since $K$ is infinite, every element of $U$ is chosen. Moreover, if $k \in K$ then $\max \left\{r_{k},\left|s_{k}\right|\right\} \leq k$, since only $k-1$ elements of $U$ have previously been chosen.

For each $j$ define $\tau_{j}^{\prime}=\left[h_{j}^{\prime}\right]$, where $h_{j}^{\prime}(p)=\operatorname{det}_{p}(k, j)$ whenever $0=$ $h_{j}(p)<\operatorname{det}_{p}(k, j)<h_{k}(p)=\infty$ for some $j<k \notin K$ with $\tau_{k}$ very large, and define $h_{j}^{\prime}(p)=h_{j}(p)$ otherwise. Note that $\tau_{j}^{\prime} \geq \tau_{j}$ and $h_{j}^{\prime}(p)=\infty$ iff $h_{j}(p)=\infty$.

By Theorem 2.5 , it is sufficient to prove that $T^{\prime}=\left(\tau_{1}^{\prime}, \tau_{2}^{\prime}, \ldots\right)$ is admissible relative to the chosen ordering of $U$. Fix $j$ and let $m=$ $\max \left\{r_{j},\left|s_{j}\right|\right\}$.

Let $P_{1}=\left\{p \mid 0=h_{j}^{\prime}(p)<\operatorname{det}_{p}(k, j)<h_{k}^{\prime}(p)=\infty\right.$ for some $\left.k \in K\right\}$. If $p=p_{i} \in P_{1}$ then $p_{i}$ divides $\operatorname{det}(k, j)$ while $\operatorname{det}(k, j) \leq 2 m k \leq 4 m i$, since $k \in K$ and $h_{k}\left(p_{i}\right)=\infty$ implies that $k \leq 2 i$. By Lemma 0.1.b, $p_{i}>4 m i$ for sufficiently large $i$, so that $P_{1}$ is finite.

Next let $P_{2}=\left\{p \mid h_{j}^{\prime}(p) \neq \operatorname{det}_{p}(j, k)<h_{k}^{\prime}(p)=\infty, \tau_{k}\right.$ not very large, $j<k \notin K\}$. By the choice of $u_{k}=\left(r_{k}, s_{k}\right) \in U, j>n_{k}$ for each such $k$. Assume that $P_{2}$ is infinite. Then there are infinitely many $j<k \notin K$ with $j>n_{k}, \inf \left\{p \mid h_{k}(p)=\infty\right\}>j$ (noting that for each $p$ there is at most one $i$ with $\left.h_{i}(p)=\infty\right)$, and $h_{j}(p) \neq \operatorname{det}_{p}(j, k)<h_{k}(p)=\infty$ for some $p \in$ $P_{2}$. For each such $k$, there is $1 \leq i \neq l \leq j$ with $0<\operatorname{det}_{p}(i, l)<h_{k}(p)=$ $\infty$ for some $p$, otherwise $j \leq n_{k}$ by the definition of $u_{k}$. But

$$
\left\{p \mid \operatorname{det}_{p}(i, l)>0 \text { for some } 1 \leq i \neq l \leq j\right\}
$$

is finite, which is a contradiction.

Finally, $P_{3}=\left\{p \mid h_{j}^{\prime}(p) \neq \operatorname{det}_{p}(j, k)<h_{k}^{\prime}(p)=\infty\right.$ for some $j<k \notin$ $K, \tau_{k}$ very large $\}$ is empty by the definition of $h_{j}^{\prime}$. Thus $P_{1} \cup P_{2} \cup P_{3}$ is finite so that $T^{\prime}$ is admissible.

(b) Note that $T_{0} \cup\left\{\tau_{j} \mid \tau_{j}\right.$ very large $\}$ generates an infinite subsequence of $T$ with no snarls in $T$. Now apply the constructions of (a), noting that if $k \notin K$ then $\tau_{k}$ is not very large so that $h_{j}^{\prime}=h_{j}$ for each $j$.

Corollary 2.10. Let $S$ be a set of types with $\inf \left\{\tau, \tau^{\prime}\right\}=\operatorname{type}(Z)$ whenever $\tau, \tau^{\prime} \in S$ with $\tau \neq \tau^{\prime}$. Assume that $\{\tau \in S \mid \tau$ is very large $\}$ has no snarls in $S$. 
(a) There is a rank-2 group $A$ with $\operatorname{typeset}(A)=S$ iff either type $(Z) \in$ $S$ or else $S$ has an infinite subset with no snarls in $S$.

(b) There is a completely anisotropic rank-2 $A$ with typeset $(A)=S$ iff $S$ has an infinite subset with no snarls in $S$.

Proof. (a) $(\Rightarrow)$ Let $T_{A}=\left(\tau_{1}, \tau_{2}, \ldots\right)$. By Theorem 1.4, $T_{A}$ has an infinite subsequence with no snarls in $T_{A}$. If $S=\left\{\tau_{i} \mid i \geq 1\right\}=\operatorname{typeset}(A)$ does not have an infinite subset with no snarls in $S$ then some $\tau_{i}$ must be repeated in $T_{A}$. But $\operatorname{IT}(A)=\operatorname{type}(Z)$ is the only type in $T_{A}$ that may be repeated so that type $(Z) \in S$.

$(\Leftarrow)$ If $S$ has an infinite subset with no snarls in $S$ define $T=$ $\left(\tau_{1}, \tau_{2}, \ldots\right)$ where $S=\left\{\tau_{l} \mid i \geq 1\right\}$. Otherwise type $(Z) \in S$, and in this case define $T=\left(\tau_{1}^{\prime}, \tau_{2}^{\prime}, \ldots\right)$ where $\tau_{2,-1}^{\prime}=\tau_{i}, \tau_{2 i}^{\prime}=\operatorname{type}(Z)$ for $i \geq 1$ if $S$ is infinite. If $S=\left\{\tau_{1}, \tau_{2}, \ldots, \tau_{n}\right\}$ is finite define $\tau_{2 l}^{\prime}=\operatorname{type}(Z), \tau_{2 i-1}^{\prime}=\tau_{l}$ for $1 \leq i \leq n$ and $\tau_{i}^{\prime}=\tau_{2 i-1}^{\prime}=\operatorname{type}(Z)$ for $i>n$. For each of the above cases, $T$ has an infinite subset with no snarls in $T$. By Theorem 2.9 there is a rank-2 group $A$ with $T_{A} \approx T$ so that typeset $(A)=S$.

(b) is a consequence of (a) and the fact that $A$ is completely anisotropic iff $T_{A}$ has no repetitions.

Corollary 2.11. Let $S_{1}=\left\{\tau_{1}, \tau_{2}, \ldots\right\}$ be a set of types with $\inf \left\{\tau_{i}, \tau_{j}\right\}$ $=\operatorname{type}(Z)$ whenever $i \neq j$, and assume that $\left\{\tau_{j} \mid \tau_{j}\right.$ is very large $\}$ has no snarls in $S_{1}$. Let $S_{2}=\left(\sigma_{1}, \sigma_{2}, \ldots\right)$ be another set of types. Then there is a rank-2 group $A$ with $\operatorname{typeset}(A)=S_{1}$ and $\operatorname{cotypeset}(A)=S_{2}$ if and only if

(a) There is a type $\sigma_{0}$ such that $\sigma_{0}=\sup \left\{\sigma_{i}, \sigma_{j}\right\}$ for $i \neq j$;

(b) $\tau_{i} \leq \sigma_{0}$ for each $i$

(c) $\sigma_{l}=\sigma_{0}-\tau_{i}$ for each $i$; and

(d) Either type $(Z) \in S_{1}$ or else $S_{1}$ has an infinite subset with no snarls in $S_{1}$.

Proof. $(\Rightarrow$ ) Apply Proposition 1.1 and Corollary 2.10.

$(\Leftarrow)$ In view of $(\mathrm{d})$, Theorem 2.9 can be applied to obtain a group $B$ such that $\operatorname{typeset}(B)=S_{1}$. Furthermore, $B$ can be assumed to satisfy $\mathrm{OT}(B) \leq \sigma_{0}$ by (b) and Lemma 2.2. By Theorem 2.6, there is a rank-2 group $A$ such that $\operatorname{typeset}(A)=\operatorname{typeset}(B)$ and $\mathrm{OT}(A)=\sigma_{0}$. By (c) and Proposition 1.1(e), $\operatorname{cotypeset}(A)=\left\{\sigma_{1}, \sigma_{2}, \ldots\right\}=S_{2}$.

EXAMPLE 2.12. Let $\tau_{l}$ for $i \geq 1$ be defined as in Example 1.5. Let $S=\left\{\tau_{l} \mid i \geq 1\right\} \cup\{\operatorname{type}(Z)\}$. Then there is a rank-2 group $A$ with typeset $(A)=S$, by Corollary 2.10.a. On the other hand, by Corollary $2.10 . \mathrm{b}$ there is no completely anisotropic rank-2 group $A$ with typeset $(A)$ $=S$ (compare Example 1.5). 
EXAMPLE 2.13. (Ito [9].) Let $\tau_{1}, \tau_{2}, \ldots$ be given by $\tau_{l}=\left[h_{i}\right]$, where $h_{1}=(0,1,0,1,0,1, \ldots) ; \quad h_{2}=(\infty, 0,0, \ldots) ; h_{3}=(0, \infty, 0, \ldots) ; h_{4}=$ $(0,0, \infty, \ldots) ; \ldots$. Let $S=\left\{\tau_{i} \mid i \geq 1\right\}$. Then $\left\{\tau_{2 i} \mid i \geq 1\right\}$ is an infinite subset of $S$ with no snarls in $S$. By Corollary 2.10(b), there is a completely anisotropic rank-2 group $A$ with typeset $(A)=S$. Similarly, there is a completely anisotropic rank-2 group $A$ with $\operatorname{typeset}(A)=S \cup\{\operatorname{type}(Z)\}$.

COROLlaRY 2.14. Let $S=\left\{\tau_{i} \mid i \geq 1\right\}$ be a set of types with $\tau_{0}=$ $\inf \left\{\tau_{i}, \tau_{j}\right\}$ whenever $i \neq j$.

(a) (Beaumont-Pierce [3]) If $S$ is finite and $\tau_{0} \in S$ then there is a rank-2 group $A$ with typeset $(A)=S$.

(b) (Ito [9]) If there is $h_{l} \in \tau_{i}$ for $i \geq 0$ with $h_{0}=\inf \left\{h_{i}, h_{j}\right\}$ for each $i \neq j$ then there is a rank-2 group $A$ with $\operatorname{typeset}(A)=S$ and $\mathrm{OT}(A)=$ $\left[\sup \left\{h_{i} \mid i \geq 1\right\}\right]$.

Proof. By Lemma 1.3, it suffices to assume that $\tau_{0}=\operatorname{type}(Z)$. In either case $S$ has no snarls in $S$. Now apply Corollary 2.10 to get a rank-2 group $A$ with typeset $(A)=S$. This group is constructed via Theorem 2.9 so that $\mathrm{OT}(A) \leq\left[\sup \left\{h_{l} \mid i \geq 1\right\}\right]$. By Theorem $2.6, A$ may be chosen with $\mathrm{OT}(A)=\sup \left[\left\{h_{i} \mid i \geq 1\right\}\right]$.

The next example shows that the hypothesis of Corollary 2.10 that $\{\tau \in S \mid \tau$ is very large $\}$ has no snarls in $S$ is not necessary.

EXAMPLE 2.15. There is a rank-2 group $A$ such that $\operatorname{IT}(A)=\operatorname{type}(Z)$ and $\{\tau \mid \tau \in \operatorname{typeset}(A)$ and $\tau$ very large $\}$ is infinite with infinitely many snarls in typeset $(A)$.

Proof. Let $S=\left\{\tau_{i} \mid i \geq 1\right\}$ where $\tau_{i}=\left[h_{i}\right]$ and $h_{i}$ is defined by:

$$
\begin{aligned}
& h_{1}=(1,1,1, \ldots, \infty, \infty, \infty, \ldots, 0,0,0, \ldots, 0,0,0, \ldots, 0,0,0, \ldots) \\
& h_{2}=(\infty, 0,0, \ldots, 0,0,0, \ldots, 1,1,1, \ldots, \infty, \infty, \infty, \ldots, 0,0,0, \ldots) \\
& h_{3}=(0, \infty, 0, \ldots, 0,0,0, \ldots, \infty, 0,0, \ldots, 0,0,0, \ldots, 1,1,1, \ldots), \text { etc. }
\end{aligned}
$$

Apply Theorem 2.9(a) to obtain a rank-2 group $A$ with typeset $(A)=\left\{\tau_{i}^{\prime} \mid i\right.$ $\geq 1\}, \tau_{l}^{\prime} \geq \tau_{l}$ for each $i$, and $\{\tau \mid \tau \in \operatorname{typeset}(A)$ and $\tau$ very large $\}$ is infinite with infinitely many snarls in $\operatorname{typeset}(A)$.

\section{Realization of cotypesets.}

THEOREM 3.1 (Vinsonhaler-Wickless [12]). Let $S=\left\{\sigma_{1}, \sigma_{2}, \ldots\right\}$ be a set of types with $\sigma_{0}=\sup \left\{\sigma_{i}, \sigma_{j}\right\}$ for each $i \neq j$ and $\sigma_{0} \in S$ if $S$ is finite.

(a) There is $s_{i} \in \sigma_{i}$ for $i \geq 0$ such that $s_{0}=\max \left\{s_{i}, s_{j}\right\}$ if $i \neq j$. 
(b) There is a rank-2 group $A$ with $\operatorname{cotypeset}(A)=S$, OT $(A)=\sigma_{0}$, $\operatorname{IT}(A)=\left[\inf \left\{s_{l} \mid i \geq 1\right\}\right]$, and $\operatorname{typeset}(A)=\left\{\sigma_{0}-\sigma_{l}+\operatorname{IT}(A) \mid i \geq 1\right\}$.

Proof. The following proof is a simplification of the arguments given in Vinsonhaler-Wickless [12].

(a) Given $s_{0}, s_{1}, \ldots, s_{n-1}$ with $s_{l} \in \sigma_{i}$ for $0 \leq i \leq n-1$ and $s_{0}=$ $\max \left\{s_{i}, s_{j}\right\}$ for $1 \leq i \neq j \leq n-1$ choose $s_{n} \in \sigma_{n}$ with $s_{0}=\sup \left\{s_{l}, s_{n}\right\}$ for $1 \leq i \leq n-1$.

(b) Define $t_{0}^{\prime}=\min \left\{s_{l} \mid i \geq 1\right\}, \tau_{0}^{\prime}=\left[t_{0}^{\prime}\right]$, and $\gamma_{l}=\sigma_{t}-\tau_{0}^{\prime}$ for $i \geq 0$. Note that $\gamma_{i}=\left[s_{l}-t_{0}^{\prime}\right]$ for each $i \geq 0$. Now $\Gamma=\left\{\gamma_{1}, \gamma_{2}, \ldots\right\}$ with $\gamma_{0}=$ $\sup \left\{\gamma_{i}, \gamma_{j}\right\}$ if $i \neq j$ and $\gamma_{0} \in \Gamma$ if $\Gamma$ is finite. Define $\tau_{i}=\gamma_{0}-\gamma_{l}$ for $i \geq 0$.

The next step is to show that there is a rank-2 group $B$ with $\operatorname{typeset}(B)=\left\{\tau_{l} \mid i \geq 1\right\}$ and $\operatorname{cotypeset}(B)=\Gamma$. For each $i$, let $t_{t}=\left(s_{0}-\right.$ $\left.t_{0}^{\prime}\right)-\left(s_{l}-t_{0}^{\prime}\right) \in \tau_{l}=\gamma_{0}-\gamma_{l}$. Note that:

(i) if $t_{l}(p)=\infty$ then $s_{0}(p)=\infty, t_{0}^{\prime}(p)<\infty$, and $s_{l}(p)<\infty$.

(ii) if $0<t_{l}(p)<\infty$ then $s_{0}(p)<\infty$.

(iii) $t_{i}(p)=s_{0}(p)-s_{i}(p)$.

(iv) $\inf \left\{t_{l}, t_{j}\right\}=0$ whenever $i \neq j$.

By (iv) and Corollary 2.14.b there is a rank-2 group $B$ with typeset $(B)$ $=\left\{\tau_{i} \mid i \geq 1\right\}, \operatorname{OT}(B)=\left[\sup \left\{t_{l} \mid i \geq 1\right\}\right]$, and $\operatorname{IT}(B)=\tau_{0}=\operatorname{type}(Z)$. By (iii), $\sup \left\{t_{i} \mid i \geq 1\right\}=s_{0}-t_{0}^{\prime}$ so that OT $(B)=\gamma_{0}$. Therefore, $\operatorname{cotypeset}(B)$ $=\left\{\gamma_{0}-\tau_{i} \mid i \geq 1\right\}$ by Proposition 1.1.e.

Furthermore, $\gamma_{0}-\tau_{l}=\gamma_{l}$ for each $i \geq 0$. To see this, note that $\gamma_{0}-\tau_{i}$ $=\left[\left(s_{0}-t_{0}^{\prime}\right)-\left(s_{0}-s_{l}\right)\right]$, by (iii), and $\gamma_{l}=\left[s_{l}-t_{0}^{\prime}\right]$. The only non-trivial case is $t_{0}^{\prime}(p) \leq s_{l}(p)<s_{0}(p)=\infty$, in which case $s_{j}(p)=s_{0}(p)$ for $j \neq i$ (by (a)), $t_{0}^{\prime}(p)=s_{l}(p)$, and $\left(s_{0}(p)-t_{0}^{\prime}(p)\right)-\left(s_{0}(p)-s_{l}(p)\right)=0=$ $s_{l}(p)-t_{0}^{\prime}(p)$. Consequently, $\operatorname{cotypeset}(B)=\left\{\gamma_{l} \mid i \geq 1\right\}$.

By Lemma 1.3, there is a rank-2 group $A$ with typeset $(A)=\left\{\tau_{l}+\tau_{0}^{\prime} \mid i\right.$ $\geq 1\}, \operatorname{IT}(A)=\tau_{0}^{\prime}, \operatorname{cotypeset}(A)=\left\{\gamma_{1}+\tau_{0}^{\prime} \mid i \geq 1\right\}=S$, and $\operatorname{OT}(A)=\gamma_{0}$ $+\tau_{0}^{\prime}=\sigma_{0}$. Finally, $\tau_{l}+\tau_{0}^{\prime}=\sigma_{0}-\sigma_{l}+\tau_{0}^{\prime}$ by (iii).

COROllary 3.2. Let $S=\left\{\sigma_{1}, \sigma_{2}, \ldots\right\}$ be a set of types. There is a rank-2 group $A$ with $\operatorname{cotypeset}(A)=S$ iff there is $\sigma_{0}=\sup \left\{\sigma_{l}, \sigma_{j}\right\}$ whenever $i \neq j$ and $\sigma_{0} \in S$ if $S$ is finite.

REMARK. Vinsonhaler-Wickless [12] have given necessary and sufficient conditions for a set of types to be the cotypeset of a finite rank torsion free group, with Corollary 3.2 as a special case.

4. Locally completely decomposable groups. A finite rank torsion free group $A$ is locally completely decomposable if $A_{p}=Z_{p} \otimes_{Z} A$ is the 
direct sum of a free $Z_{p}$-module and a divisible $Z_{p}$-module for each prime $p$, where $Z_{p}$ is the localization of $Z$ at $p$.

Let $A$ be a finite rank torsion free group. Recall that typeset $(A)=$ $\{\operatorname{type}(X) \mid X$ is a pure rank-1 subgroup of $A\}$ and $\operatorname{cotypeset}(A)=$ $\{\operatorname{type}(Y) \mid Y$ is a rank-1 torsion free quotient of $A\}$.

THEOREM 4.1. Assume that $A$ is a finite rank torsion free group.

(a) There is a finite rank torsion free locally completely decomposable group $B$ with $B \subseteq A, A / B$ torsion, and typeset $(B)=\operatorname{typeset}(A)$.

(b) (Vinsonhaler-Wickless [12]). There is a finite rank torsion free locally completely decomposable group $B$ with $A \subseteq B, B / A$ torsion, and $\operatorname{cotypeset}(A)=\operatorname{cotypeset}(B)$.

(c) Further assume that $\operatorname{rank}(A)=2, \operatorname{typeset}(A)=\left\{\tau_{l} \mid i \geq 1\right\}, h_{l} \in \tau_{t}$ for each $i$ and $s_{0} \in \mathrm{OT}(A)$. Then $A$ is locally completely decomposable iff whenever $s_{0}(p)=\infty$ then $h_{i}(p)=\infty$ for some $i$.

Proof. (a) Let $A_{1}, A_{2}, \ldots$ be a listing of the pure rank-1 subgroups of $A$, let $p_{i}$ be the $i$ th prime and choose a free subgroup $F$ of $A$ with $A / F$ torsion.

Define $B_{p_{t}}=F_{p_{i}}+d\left(A_{p_{t}}\right)+\left(A_{1}\right)_{p_{t}}+\cdots+\left(A_{i}\right)_{p_{t}}$ where $d\left(A_{p}\right)$ is the maximal divisible $Z_{p}$-submodule of $A_{p}$. Define $B=\cap_{p} B_{p}$. Then $F=\bigcap_{p} F_{p} \subseteq B \subseteq A=\bigcap_{p} A_{p}$ and $A / B$ is torsion. Let $X=A_{i}$. Then $X_{p}$ $\subseteq B_{p}$ for almost all $p$. If $X_{p} \simeq Q$ then $X_{p} \subseteq d\left(A_{p}\right) \subseteq B_{p}$. Otherwise, $X_{p} /\left(X_{p} \cap B_{p}\right)$ is finite. Hence $X / X \cap B$ is finite since $(X / X \cap B)_{p} \cong$ $X_{p} / X_{p} \cap B_{p}=0$ for almost all $p$ and $X_{p} /\left(X_{p} \cap B_{p}\right)$ is finite otherwise. Consequently, $X \cong X \cap B$ and so $\operatorname{typeset}(A)=\operatorname{typeset}(B)$. Finally, $B$ is locally completely decomposable since for each $p, B_{p} / d\left(B_{p}\right)$ is a finitely generated free $Z_{p}$-module.

(b) The following is a rank-2 version of the proof in VinsonhalerWickless [13]. Define

$$
B=\bigcap\left\{f^{-1} f(A) \mid f \in \operatorname{Hom}(Q A, Q)\right\} \subseteq Q A=Q \otimes_{Z} A
$$

where $A$ is regarded as a subgroup of $Q A$. Then $A \subseteq B \subseteq Q A$ and $B / A$ is torsion. Suppose that $g(A)=Y \subseteq Q$ for some $g: A \rightarrow Q$. Then $g: Q A \rightarrow Q$ and $g(A) \subseteq g(B) \subseteq g\left(g^{-1} g(A)\right)=g(A)$. Conversely, if $g(B)=Y \subseteq Q$ for some $g: B \rightarrow Q$ then $g: Q A \rightarrow Q$ since $Q A=Q B$ and $g(A) \subseteq g(B) \subseteq$ $g\left(g^{-1} g(A)\right)=g(A)$. Consequently, $\operatorname{cotypeset}(A)=\operatorname{cotypeset}(B)$, noting that each rank-1 torsion free group is isomorphic to a subgroup of $Q$.

To show that $B$ is locally completely decomposable suppose that $B_{p}=X \oplus Y$ where $X$ has no rank-1 summands and $Y$ is the direct sum of a free and a divisible $Z_{p}$-module. Let $0 \neq f \in \operatorname{Hom}(Q A, Q)$. If $f(X) \neq 0$, 
then $f(X)=Q=f\left(B_{p}\right)$, since otherwise $f(X) \simeq Z_{p}$ and $X$ has no rank-1 summands. But $\operatorname{Ker} f$ is divisible so $B_{p} \subseteq f^{-1} f\left(B_{p}\right)=\operatorname{Ker} f \oplus H$ where $f(H)=f\left(B_{p}\right)=Q$. Thus $H$ is divisible so that $f^{-1} f\left(B_{p}\right)=f^{-1} f\left(A_{p}\right)=$ $Q A \supseteq Q X$ in this case. Now assume that $f(X)=0$. Then $Q X \subseteq \operatorname{Ker} f \subseteq$ $f^{-1} f\left(A_{p}\right)$. Thus, $Q X \subseteq f^{-1} f\left(A_{p}\right)$ for all $f \in \operatorname{Hom}(Q A, Q)$. But

$$
\begin{aligned}
B_{p} & =\left(\bigcap\left\{f^{-1} f(A) \mid f \in \operatorname{Hom}(Q A, Q)\right\}\right)_{p} \\
& =\bigcap\left\{f^{-1} f\left(A_{p}\right) \mid f \in \operatorname{Hom}(Q A, Q)\right\} \supseteq Q X
\end{aligned}
$$

so that $Q X=X$ is divisible. Since $X$ has no rank-1 summands, $X=0$ as desired.

(c) $(\Rightarrow)$ Let $A_{p}=X_{1} \oplus X_{2}$. Then there are pure rank-1 subgroups $A_{l}$ of $A$ with $\left(A_{i}\right)_{p}=X_{i}$. If $s_{0}(p)=\infty$ then $\left(A / A_{i}\right)_{p} \approx Q$ for some $i$, say $i=1$. But $\left(A / A_{1}\right)_{p} \simeq A_{p} /\left(A_{1}\right)_{p} \simeq X_{2}$ so that $X_{2} \simeq Q$ and $A_{2}$ is $p$-divisible. Therefore, for some $k, \tau_{k}=\operatorname{typeset}\left(A_{2}\right)$ and if $h_{k} \in \tau_{k}$ then $h_{k}(p)=\infty$.

$(\Leftarrow)$ Assume that $s_{0}(p)<\infty$ and that $A_{i}$ is a pure rank-1 subgroup of $A$. Then $0 \rightarrow\left(A_{i}\right)_{p} \rightarrow A_{p} \rightarrow\left(A / A_{i}\right)_{p} \rightarrow 0$ is exact with $\left(A / A_{i}\right)_{p} \simeq Z_{p}$ since type $\left(A / A_{1}\right) \leq \mathrm{OT}(A)$. Thus $A_{p} \simeq Z_{p} \oplus\left(A_{l}\right)_{p}$ is completely decomposable. Now assume that $s_{0}(p)=\infty=h_{i}(p)$. Let $A_{i}$ be a pure rank-1 subgroup of $A$ with $\tau_{i}=\operatorname{type}\left(A_{i}\right)$. Then $\left(A_{i}\right)_{p} \simeq Q$ so that $A_{p} \simeq Q \oplus$ $\left(A / A_{i}\right)_{p}$ is completely decomposable as desired.

REMARK. In view of Theorem 4.1(c), each rank-2 group constructed in Theorem 2.5 is locally completely decomposable, noting that the $h_{i} \in \tau_{l}$ in this construction are chosen to satisfy (a) of Lemma 2.1.

COROllary 4.2. Let $S_{1}=\left\{\tau_{1}, \tau_{2}, \ldots\right\}$ be a set of types with $\inf \left\{\tau_{i}, \tau_{j}\right\}$ $=\operatorname{type}(Z)$ whenever $i \neq j$ and assume that $\left\{\tau_{j} \mid \tau_{j}\right.$ very large $\}$ has no snarls in $S_{1}$. Let $S_{2}=\left\{\sigma_{1}, \sigma_{2}, \ldots\right\}$ be another set of types. Then there is a rank-2 locally complete decomposable group $A$ with $\operatorname{typeset}(A)=S_{1}$ and $\operatorname{cotypeset}(A)=S_{2}$ iff

(a) There is a type $\sigma_{0}$ such that $\sigma_{0}=\sup \left\{\sigma_{l}, \sigma_{j}\right\}$ for $i \neq j$;

(b) $\tau_{i} \leq \sigma_{0}$ for each $i$;

(c) $\sigma_{i}=\sigma_{0}-\tau_{l}$ for each $i$;

(d) Either type $(Z) \in S_{1}$ or else $S_{1}$ has an infinite subset with no snarls in $S_{1}$;

(e) If $s_{0} \in \sigma_{0}, h_{i} \in \tau_{i}$ for each $i$, and $s_{0}(p)=\infty$ then $h_{i}(p)=\infty$ for some $i$.

Proof. A consequence of Corollary 2.11, Theorem 4.1(c), and the preceding remark. 


\section{Open questions.}

(5.1) Is it true that Corollaries 2.10 and 2.11 are true without the hypothesis that $\{\tau \in S \mid \tau$ is very large $\}$ has no snarls in $S$ ?

As noted earlier, Example 2.15 shows that this hypothesis is not necessary. In fact, it is unknown whether or not the set $S$ of types in Example 2.15 may be realized as the typeset of a rank-2 group.

The construction of Theorem 2.9 uses Lemma 2.8. Consequently, some strengthened, possibly infinite, version of Lemma 2.8 would be needed to make the construction of Theorem 2.9 work without the hypothesis that $\left\{\tau_{j} \mid \tau_{j}\right.$ very large $\}$ has no snarls in $T$.

(5.2) Are the results of the paper true for modules over an arbitrary principal ideal domain?

The results of this paper use a version of the prime number theorem (Lemma 0.1(b)) which is not applicable for arbitrary principal ideal domains. De Munter-Kuyl [4], claims that Ito's Theorem (Corollary $2.14(b))$ is true for arbitrary principal ideal domains. However, the construction is incorrect, even in the case of groups. For example, the construction fails for a set of types $=\left\{\tau_{l} \mid i \geq 1\right\}$ where $\tau_{i}=\left[h_{l}\right]$ and $h_{1}=(\infty, 0, \ldots), h_{2}=(0, \infty, 0, \ldots), h_{3}=(0,0, \infty, \ldots), \ldots$, even though Ito's theorem is true for groups.

The answer to (5.2) may depend on:

(5.3) Can the results of this paper be proved without appealing to some version of the prime number theorem?

\section{REFERENCES}

[1] D. Arnold, Pure Subgroups of Finite Rank Completely Decomposable Groups, Proceedings of Oberwolfach Abelian group theory conference, Springer-Verlag Lecture Notes in Mathematics 874 (1981), 1-28.

[2] _ Finite Rank Torsion Free Groups and Rings, Springer-Verlag Lecture Notes in Mathematics \# 931, Berlin, Heidelberg, New York, 1982.

[3] R. Beaumont and R. Pierce, Torsion free groups of rank two, Mem. Amer. Math. Soc., 38 (1961).

[4] L. De Munter-Kuyl, $A$ note on the existence of torsion-free modules with a prescribed type-set, Proc. Amer. Math. Soc., 80 (1980), 531-532.

[5] D. Dubois, Applications of analytic number theory to the study of typesets of torsion free abelian groups I, Publ. Math., 12 (1965), 59-63.

[6] Applications of analytic number theory to the study of typesets of torsion free abelian groups II, Publ. Math., 13 (1966), 1-8.

[7] L. Fuchs, Infinite Abelian Groups, Vol. II, Academic Press, New York, 1973.

[8] G. Hardy and E. Wright, An Introduction to the Theory of Numbers, Oxford Univ. Press, Oxford, 1979.

[9] R. Ito, On type-sets of torsion free abelian groups of rank two, Proc. Amer. Math. Soc., 48 (1975), 39-42.

[10] J. Koehler, Some torsion free rank two groups, Acta. Sci. Math. Szeged, 25 (1964), $186-190$. 
[11] P. Schultz, The typeset and cotypeset of a rank two abelian group, Pacific J. Math., 70 (1978), 503-517.

[12] C. Vinsonhaler and W. Wickless, The cotypeset of a torsion free abelian group of rank two, Proc. Amer. Math. Soc., 84 (1982), 467-473.

[13] _ The cotypeset of a torsion free abelian group of finite rank, J. Algebra, 83 No. 2 (1983), 380-386.

[14] R. Warfield, Jr., Homomorphisms and duality of torsion free groups, Math. Z., 107 (1968), 189-200.

Received December 7, 1982. First author's research supported in part by NSF Grant \# MCS-8003060.

UNIVERSITY OF CONNECTICUT

STORRS, CT 06268 



\section{PACIFIC JOURNAL OF MATHEMATICS \\ EDITORS}

DONALD BABBITt (Managing Editor)

University of California

Los Angeles, CA 90024

J. Dugundu

University of Southern California

Los Angeles, CA 90089-1113

R. FINN

Stanford University

Stanford, CA 94305

HERMANN FLASCHKA

University of Arizona

Tucson, AZ 85721
C. C. MOORE

University of California

Berkeley, CA 94720

ARTHur Ogus

University of California

Berkeley, CA 94720

Hugo RossI

University of Utah

Salt Lake City, UT 84112

H. SAMELSON

Stanford University

Stanford, CA 94305

ASSOCIATE EDITORS

R. ARENS

E. F. BECKENBACH

B. H. NeumanN (1906-1982)

F. WOLF

K. YoshidA

\section{SUPPORTING INSTITUTIONS}

UNIVERSITY OF ARIZONA

UNIVERSITY OF BRITISH COLUMBIA

UNIVERSITY OF OREGON

CALIFORNIA INSTITUTE OF TECHNOLOGY

UNIVERSITY OF CALIFORNIA

MONTANA STATE UNIVERSITY

UNIVERSITY OF SOUTHERN CALIFORNIA

UNIVERSITY OF NEVADA, RENO

STANFORD UNIVERSITY

UNIVERSITY OF HAWAII

NEW MEXICO STATE UNIVERSITY

UNIVERSITY OF TOKYO

UNIVERSITY OF UTAH

OREGON STATE UNIVERSITY

WASHINGTON STATE UNIVERSITY

UNIVERSITY OF WASHINGTON 


\section{Pacific Journal of Mathematics}

Vol. 114, No. $1 \quad$ May, 1984

David Marion Arnold and Charles Irvin Vinsonhaler, Typesets and cotypesets of rank-2 torsion free abelian groups $\ldots \ldots \ldots \ldots \ldots \ldots \ldots 1$

Duncan Alan Buell and Richard Howard Hudson, Solutions of certain

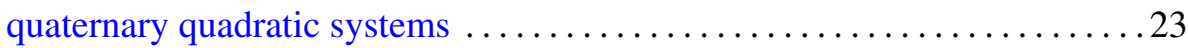

Hans Delfs and Manfred Knebusch, Separation, retractions and homotopy

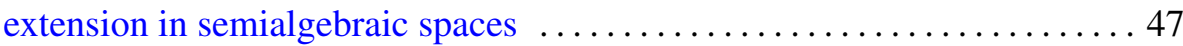

I. Erdélyi and Sheng-Wang Wang, A spectral duality theorem for closed

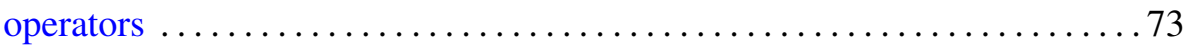

Theodore William Gamelin, Weak compactness of representing measures for $R(K)$

Kenneth R. Goodearl and T. H. Lenagan, Krull dimension of

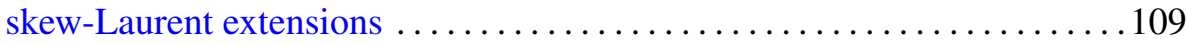

Daniel Joseph Gross, Compact quotients by $\mathbf{C}^{*}$-actions . . . . . . . . . . . 149

Goo Ishikawa, Satoshi Koike and Masahiro Shiota, Critical value sets of

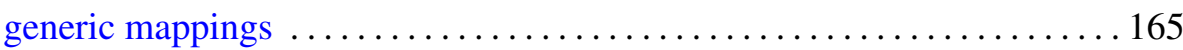

Hong Oh Kim, Derivatives of Blaschke products $\ldots \ldots \ldots \ldots \ldots \ldots \ldots \ldots \ldots$

Erhard Luft and Denis Karmen Sjerve, 3-manifolds with subgroups

$Z \oplus Z \oplus Z$ in their fundamental groups $\ldots \ldots \ldots \ldots \ldots \ldots \ldots \ldots \ldots \ldots \ldots \ldots$

George Clifford Nelson, Boolean powers, recursive models, and the Horn

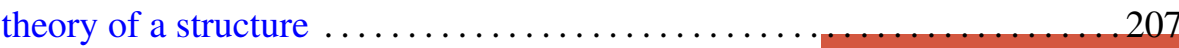

W. J. Phillips, Flow under a function and discrete decomposition of properly infinite $W^{*}$-algebras

Teodor C. Przymusiński, A solution to a problem of E. Michael ... 235

Bruce Harvey Wagner, Derivations of quasitriangular algebras 243 